\title{
3D Body Scanning has Suitable Reliability: An Anthropometric Investigation for Garment Construction
}

\author{
Christopher J. PARKER, Simeon GILL, Stephen G. HAYES \\ The University of Manchester, Manchester, UK
}

DOI: $10.15221 / 17.298$ http://dx.doi.org/10.15221/17.298

\begin{abstract}
3D Body Scanning provides access to a greater breadth and depth of anthropometric data for industry applications and scientific research than at any other point in history. However, while the potential of this technology for revolutionising industrial and scientific practices has been established, little exists addressing its appropriateness in terms of reliability within recognised allowable error. To address this issue, this study investigates the degree to which 3D Body Scanning can produce reliable anthropometric measurements for use in garment construction and scientific research. 27 participants were repeatedly scanned using a SizeStream Body Scanner five consecutive times to find out the variation in reliability between instances of data capture. The variance to three Standard Deviations was then compared to established allowable error guidelines to assess suitability of the measurements attained. The main outcome of this study is that 3D Body Scanning can achieve suitable reliability to be used in garment construction, tailoring, and scientific research; providing $99.73 \%$ confidence in the suitability of extracted dimensions in $49 \%$ of the most commonly used body measurements. However, other measurements achieved less exceptional reliability, and therefore use of traditional measurement methods need to be considered carefully when generating tailored garments or comparing anthropometric data sets from 3D Body Scanners. As a consequence of this study, 3D Body Scanning shows suitable reliability for most garment construction and anthropometric research tasks.
\end{abstract}

Keywords: Reliability, 3D Body Scanning, Anthropometrics, Garment Construction

\section{Introduction}

This study explores the reliability of 3D Body Scanning as a measurement tool and whether it is suitable for use in garment construction. To confirm that 3D Body Scanning is suitable for use in tailoring, garment development, and scientific research, the reliability of technology as a measurement tool needs to be ratified through established engineering [1] and statistical [2] methodologies.

Despite the global fashion industry being worth over $\$ 212$ billion [3], 3D Body Scanning, for all its potential to drive Computer Aided Design (CAD) solutions for garment production through precise anthropometric data [4]-[6], has yet to be established as a key technology in industrial practices [7]. A crucial aspect underpinning 3D Body Scanning's application is in its ability to produce measurements that a manufacturer or designer can have as much confidence in as they can in manual methods. This claim is supported within an apparel design context by Istook and Hwang [8] who remarked that "the ability to customize garments for fit is directly tied to the availability of a comprehensive, accurate set of measurements". Academic research [7], [9] has suggested that to get suitable data for garment construction, anthropometric measurements must be both accurate and repeatable. Because of this, the ability to produce customised garments or ergonomic products for human use is tied to the appropriateness of the measurements and tools used to quantify the human body. Since measurement error is an unavoidable characteristic of anthropometrics [10], the appropriateness of such tools are tied to their ability to measure within relative boundaries. However, few which capture the variation of measurements used within product development through measurers, varied definitions, and equipment to show what is acceptable for measurement technologies.

While existing literature refers to current scanning technology to be 'accurate' [8], [11], [12], and with the right scanning method a measurement may be considered 'true' [13], it is essential to remember that reliability is relative to the engineering allowance requirements of the item being designed. This is emphasised by the exceptional work of Gordon et al. [14, p. 323] who stated that "scan-generated measurements tend to be significantly larger than those obtained by manual measurement". Further exploration of this issue was investigated in the ground-breaking work of Kuehnapfel et al. [15] who demonstrated through inter- and inter- rater investigation that 3D Body Scanning is equivalent traditional manual measurements in feasibility, reliability, and validity. However, only limited number of body measurements were included in the sample, and the results were not compared against industrial use 
allowances and this have limited context to practical application. Besides this, Han et al. [16] found that in 14 comparative measurements the difference between scan and manual measurements exceeded the ISO 20685 allowable error limits. Therefore, for use of 3D Body Scanning within scientific research, ergonomic product development, and garments for fit the reliability of the technology as measurement tools must be quantified and verified.

The aim of this study is to understand which measurements from 3D Body Scanning are suitably reliable for use in garment construction and scientific research. This is in order to establish the trust a person may place in their data when used as part of a CAD solutions for garment production. To understand the reliability of current 3D Body Scanning measurements, this study compares the precision of 90 extracted dimensions against internationally recognised allowable errors for garment construction.

This study makes the following contributions to the field of 3D Body Scanning and anthropometry:

- A method for assessing the trust one may place in the measurements taken from a 3D Body Scanner is presented, along with a recommended benchmark of minimum reliability; see Section 0.

- 90 measurements used in garment construction are presented with their reliability when taken by a 3D Body Scanner, with only 44 of those measurements shown to be suitable $99.73 \%$ of the time; see Section 0.

- 3D Body Scanning is shown to be a suitably reliable tool for garment construction and scientific research, although the measurements where caution is required are highlighted, including where manual anthropometry methods should remain the primary data capture method; see Section 0 .

\section{Theoretical Background}

In engineering, the terms appropriateness, accuracy, and reliability are of vital importance when considering if a tool is suitable for a given task. Appropriateness is the degree to which the right measurement is taken [1]. For example, the waist has multiple definitions [17], so an engineer must make sure that the suitable waist for their purpose is being taken, otherwise the measurement is not suitable. Accuracy is the closeness of measurements taken by a tool to their 'true' value [18]. For example, an engineer may want a 3D Body Scanner to take the waist at the 'Small of the Back $+3 \mathrm{~cm}$ '. But if the body scanner actually takes measurements at the 'Small of the Back $+6 \mathrm{~cm}$ ' (3cm off target), then the machine would not be deemed accurate. Both the accuracy and appropriateness of 3D Body Scanning are discussed by Gill et al. [19] within these conference proceedings, providing further insight to this topic. Reliability is the degree to which measurements can be repeatedly taken with variation occurring within pre-specified allowances, or the closeness of agreement among a set of results [18]. For example, having chosen a suitable waist measurement, and ensured that the 3D Body Scanner is calibrated to give an accurate measurement, the engineer will have to make sure that variation in measurements is within the allowable error set by garment construction theory. As reliability is the theoretical focus of this study, further explanation of its context and definition is required.

Anthropometric data has a normal (or Gaussian) distribution, as shown in Figure 1. In this distribution, $68 \%$ of all data occurs within one standard deviation $(1 \sigma)$ of the mean $(\mu)$, with $95 \%$ of all data occurring within two standard deviations of the mean $(\mu \pm 2 \sigma)$, etc. In engineering, $3 \sigma$ is a typical quota of reliability due to the high degree of associated confidence in the resulting measurement [1]. 


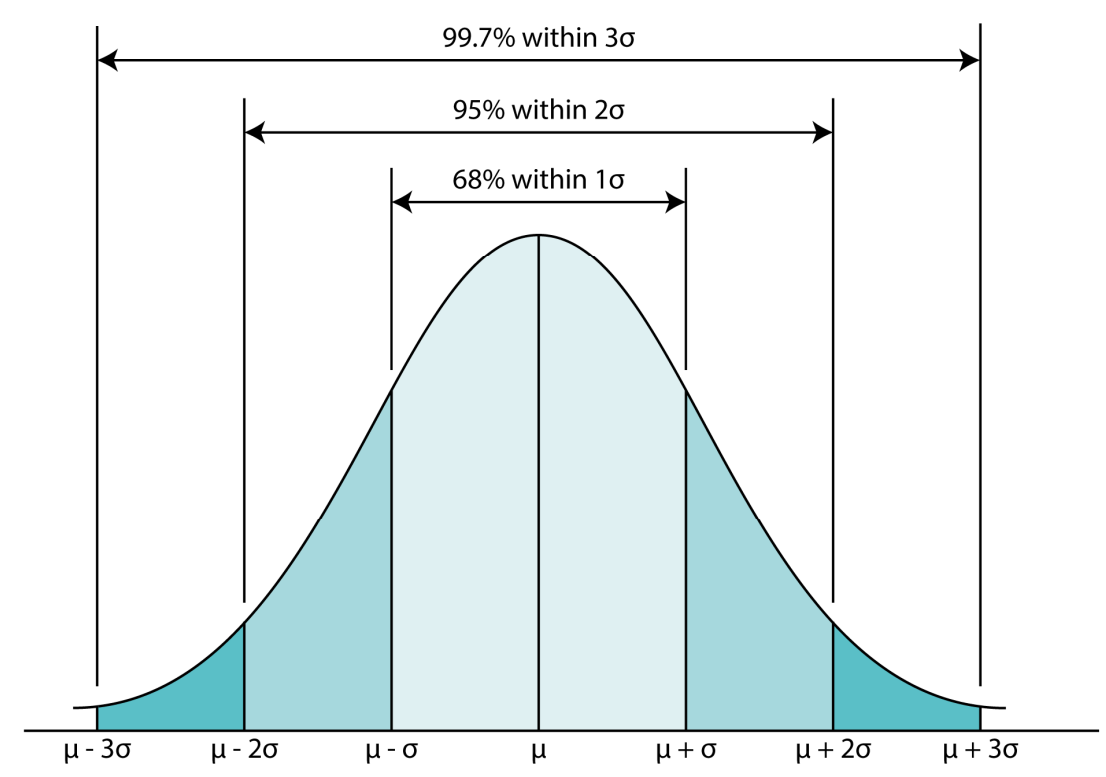

Figure 1. A Normal (Gaussian) Distribution curve

To give an example, an engineer is manufacturing rods for a new series of engines that need a length of $100 \mathrm{~mm}$, with an allowance $(A)$ of $\pm .10 \mathrm{~mm}$. She creates the rods with a machine guaranteed by the supplier to have a tolerance of $\pm .05 \mathrm{~mm}$ at the $3 \sigma$ level. Therefore, she has $99.73 \%$ confidence that the length of the rods coming out of the machine are between $100.05 \mathrm{~mm}$ and $99.95 \mathrm{~mm}(100 \mathrm{~mm} \pm .05 \mathrm{~mm})$. This, she reflects, is a very suitable tolerance for the required allowance, with less than $.03 \%$ of all manufactured rods having to be thrown away during quality control due to their lengths being too long or short. In this case, the machine is suitably reliable. However, if the engine's design had required a more precise allowance of $\pm .017 \mathrm{~mm}$, then the engineer can calculate that this new allowance is equal to her machine's tolerance at $1 \sigma$, meaning she could only produce a suitable rod $68 \%$ of the time. Here she must throw away $32 \%$ of all manufactured rods or buy a more reliable machine.

To consider Body Scanner as suitably reliable, it must be able to extract measurements from a human subject through standard body scanning protocol [20] and have the scanner's tolerance being less than or equal to the allowance. In a garment construction context, the most suitable set of allowances were established by Gordon [21], and later adopted by ISO 20685 [22] and Gordon et al. [14]. These are referred to as in their work as allowable error $( \pm A \mathrm{~mm})$ and summarised within Table 1 . The equation to judge if a 3D Body Scanning measurement is reliable is expressed in Equation 1.

\section{Equation 1. Formulae for determining reliability of a Body Scanner}

$$
3 \sigma \leq A
$$

\section{Methodology}

The scan protocol of Gill et al. [20] was used in collecting the data using a Size Stream 14 Body Scanner [23]. However, unlike standard body scan interactions where a single scan is used to capture participant's data, five scans were taken in sequence, with the participant remaining in the same scan posture between the scans. This was done to control for the independent variable of posture influencing the results [4].

27 participants (Male=15; Female=12), aged 19-60 $(M=31.1 ; S D=10.3)$ of predominantly White $(N=22)$ ethnic origin took part in the study, resulting in 135 records of data and 12,150 measurements at 90 locations on the body, analysed with SPSS [24]. As mistakes and human error [25] are not impact factors within 3D Body Scanning, mean standard deviation was calculated through Equation 2.

Equation 2. Formulae of average standard deviation

$$
\frac{\sum s t d_{i}}{n}
$$


Due to the relative low number of participants, it was not possible to remove outliers from the dataset based on the Inter Quartile Range (IQR) of Tukey [26] with a conservative power of 2.2. Because of this, the unscreened data is generalizable to the reliability of unfiltered scans as used within standard scanning protocol [20]. Reliability of body measurements was calculated as $\mu \pm 3 \sigma$ representing a precision of $99.73 \%$ confidence in the measurement [1, p. 197]. This was then compared against the rigorous allowable error $( \pm A)$ criteria of Gordon et al. [21], where rates are based on "the mean of absolute differences" between measurements taken by hand [14, p. 328].

\section{Results and Analysis}

In Table 1, the reliability of a SizeStream Body Scanner measuring 90 parts of the body, with measurements within the allowable error $( \pm A)$ highlighted in Green, showing the measurements falling within the established allowable errors for garment construction [14], [21], [22]. Table 1 shows how only 44 measurements $(48.9 \%$ of the sample) were shown to be reliable in $99.73 \%$ of measurements. A further 22 measurements (7.3\% of the sample) were shown to be reliable in $95.46 \%$ of measurements, and a final 21 measurements $(96.7 \%$ of the sample) were shown to be reliable in $68.26 \%$ of measurements. Three measurements were shown to be unreliable, failing to meet the required tolerances for garment construction.

Table 1. Reliability of Measurements (measurements in $\mathrm{mm}$, highlights show association with the column)

\begin{tabular}{|c|c|c|c|c|c|c|c|}
\hline Measurement & 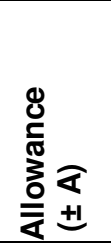 & $\stackrel{0}{-}$ & 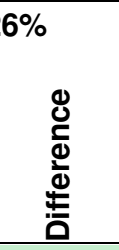 & 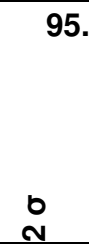 & 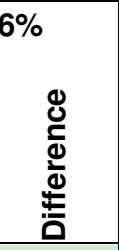 & $\begin{array}{l}0 \\
m\end{array}$ & 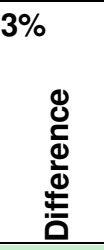 \\
\hline Shoulder Circumference & 22 & 3.46 & -18.54 & 6.91 & -15.09 & 10.37 & -11.63 \\
\hline Over Arm Circum Tape Measure & 22 & 4.55 & -17.45 & 9.10 & -12.90 & 13.66 & -8.34 \\
\hline High Hip to Small Of Back Optimised Waist & 12 & 1.24 & -10.76 & 2.49 & -9.51 & 3.73 & -8.27 \\
\hline Bust Girth With Drop Tape Measure & 15 & 2.64 & -12.36 & 5.27 & -9.73 & 7.91 & -7.09 \\
\hline Hips Two Inches Above Crotch & 12 & 1.84 & -10.16 & 3.67 & -8.33 & 5.51 & -6.49 \\
\hline Low Hip & 12 & 1.95 & -10.05 & 3.90 & -8.10 & 5.85 & -6.15 \\
\hline Seat Circum Tape Measure & 12 & 1.96 & -10.04 & 3.93 & -8.07 & 5.89 & -6.11 \\
\hline Hip Circum Tape Measure & 12 & 1.98 & -10.02 & 3.96 & -8.04 & 5.94 & -6.06 \\
\hline Axilla Chest Circumference Tape Measure & 15 & 3.12 & -11.88 & 6.25 & -8.75 & 9.37 & -5.63 \\
\hline Hips Eight Inches Down from Small of Back & 12 & 2.17 & -9.83 & 4.34 & -7.66 & 6.51 & -5.49 \\
\hline Underbust Circum Tape Measure & 16 & 3.52 & -12.48 & 7.05 & -8.95 & 10.57 & -5.43 \\
\hline Subject Height & 11 & 1.92 & -9.08 & 3.84 & -7.16 & 5.76 & -5.24 \\
\hline Hinged Upper Bust Circumference & 15 & 3.27 & -11.73 & 6.54 & -8.46 & 9.81 & -5.19 \\
\hline High Hip & 12 & 2.33 & -9.67 & 4.65 & -7.35 & 6.98 & -5.02 \\
\hline Neck Circumference & 11 & 2.02 & -8.98 & 4.04 & -6.96 & 6.06 & -4.94 \\
\hline Inseam Left & 10 & 1.82 & -8.18 & 3.65 & -6.35 & 5.47 & -4.53 \\
\hline Inseam Right & 10 & 1.83 & -8.17 & 3.66 & -6.34 & 5.49 & -4.51 \\
\hline Chest / Bust Circum Tape Measure & 15 & 3.51 & -11.49 & 7.02 & -7.98 & 10.53 & -4.47 \\
\hline Crotch Height & 10 & 1.86 & -8.14 & 3.73 & -6.27 & 5.59 & -4.41 \\
\hline Waist Circum Tape Measure & 11 & 2.37 & -8.63 & 4.75 & -6.25 & 7.12 & -3.88 \\
\hline Front Vertical Rise & 11 & 2.51 & -8.49 & 5.01 & -5.99 & 7.52 & -3.48 \\
\hline Crotch Length Full & 16 & 4.44 & -11.56 & 8.88 & -7.12 & 13.32 & -2.68 \\
\hline Calf Circumference Left & 5 & 0.80 & -4.20 & 1.61 & -3.39 & 2.41 & -2.59 \\
\hline Shoulder Circumference Height & 7 & 1.48 & -5.52 & 2.97 & -4.03 & 4.45 & -2.55 \\
\hline Min Lower Leg Girth Left & 5 & 0.89 & -4.11 & 1.78 & -3.22 & 2.67 & -2.33 \\
\hline Bicep Circumference (Right) & 8 & 1.92 & -6.08 & 3.83 & -4.17 & 5.75 & -2.25 \\
\hline
\end{tabular}




\begin{tabular}{|c|c|c|c|c|c|c|c|}
\hline Measurement & $\begin{array}{l}\mathbb{8} \\
\frac{C}{\pi} \\
\frac{\pi}{0} \\
\frac{0}{\bar{\alpha}} \pm\end{array}$ & $\stackrel{0}{-}$ & 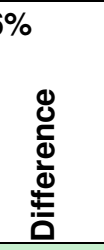 & $\stackrel{\mathfrak{n}}{\mathrm{N}}$ & 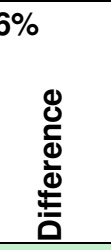 & $\stackrel{0}{m}$ & 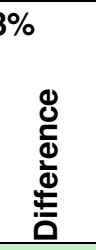 \\
\hline Min Lower Leg Girth (Right) & 5 & 0.92 & -4.08 & 1.84 & -3.16 & 2.76 & -2.24 \\
\hline Under Knee Height (Right) & 6 & 1.26 & -4.74 & 2.53 & -3.47 & 3.79 & -2.21 \\
\hline Calf Circumference (Right) & 5 & 0.94 & -4.06 & 1.89 & -3.11 & 2.83 & -2.17 \\
\hline Bicep Circumference (Left) & 8 & 1.96 & -6.04 & 3.92 & -4.08 & 5.87 & -2.13 \\
\hline Under Knee Height (Left) & 6 & 1.40 & -4.60 & 2.81 & -3.20 & 4.21 & -1.79 \\
\hline Back Neck to Shoulder Blade Tape Measure & 4 & 0.75 & -3.25 & 1.50 & -2.50 & 2.26 & -1.74 \\
\hline Seat Fold Height & 6 & 1.43 & -4.57 & 2.86 & -3.14 & 4.29 & -1.71 \\
\hline Across Chest Arm to Arm Length & 8 & 2.11 & -5.89 & 4.22 & -3.78 & 6.33 & -1.67 \\
\hline Under Knee Circumference (Left) & 4 & 0.97 & -3.03 & 1.95 & -2.05 & 2.92 & -1.08 \\
\hline Forearm Circumference (Left) & 5 & 1.33 & -3.67 & 2.65 & -2.35 & 3.98 & -1.02 \\
\hline Chin Height & 7 & 2.02 & -4.98 & 4.04 & -2.96 & 6.06 & -0.94 \\
\hline Under Knee Circumference (Right) & 4 & 1.11 & -2.89 & 2.23 & -1.77 & 3.34 & -0.66 \\
\hline Side Neck to Bust length (Right) & 8 & 2.46 & -5.54 & 4.91 & -3.09 & 7.37 & -0.63 \\
\hline Side Neck to Bust length (Left) & 8 & 2.47 & -5.53 & 4.95 & -3.06 & 7.42 & -0.58 \\
\hline Thigh Circumference (Right) & 6 & 1.84 & -4.16 & 3.68 & -2.32 & 5.52 & -0.48 \\
\hline Abdomen Circumference & 11 & 3.56 & -7.44 & 7.13 & -3.87 & 10.69 & -0.31 \\
\hline Forearm Circumference (Right) & 5 & 1.57 & -3.43 & 3.13 & -1.87 & 4.70 & -0.30 \\
\hline Thigh Circumference (Left) & 6 & 1.98 & -4.02 & 3.95 & -2.05 & 5.93 & -0.07 \\
\hline Opt Small of Back Waist & 11 & 3.77 & -7.23 & 7.54 & -3.46 & 11.31 & 0.31 \\
\hline Outer Arm Hole Circumference (Left) & 13 & 4.61 & -8.39 & 9.23 & -3.77 & 13.84 & 0.84 \\
\hline Elbow Height Waist & 11 & 4.00 & -7.00 & 8.01 & -2.99 & 12.01 & 1.01 \\
\hline Arm Hole Circumference (Right) & 13 & 5.06 & -7.94 & 10.12 & -2.88 & 15.18 & 2.18 \\
\hline Elbow Height Waist & 11 & 4.15 & -6.85 & 8.29 & -2.71 & 12.44 & 1.44 \\
\hline Outer Arm Hole Circumference (Right) & 13 & 5.17 & -7.83 & 10.34 & -2.67 & 15.50 & 2.50 \\
\hline Arm Hole Circumference (Left) & 13 & 5.20 & -7.80 & 10.39 & -2.61 & 15.59 & 2.59 \\
\hline Collar Circumference & 6 & 2.15 & -3.85 & 4.30 & -1.70 & 6.45 & 0.45 \\
\hline Back Vertical Rise & 6 & 2.51 & -3.49 & 5.01 & -0.99 & 7.52 & 1.52 \\
\hline Opt Small Waist Back Height & 4 & 1.52 & -2.48 & 3.03 & -0.97 & 4.55 & 0.55 \\
\hline Opt Small Waist Front Height & 4 & 1.52 & -2.48 & 3.03 & -0.97 & 4.55 & 0.55 \\
\hline Opt Small Waist Left Height & 4 & 1.52 & -2.48 & 3.03 & -0.97 & 4.55 & 0.55 \\
\hline Elbow Circumference Tape Measure (Right) & 4 & 1.52 & -2.48 & 3.03 & -0.97 & 4.55 & 0.55 \\
\hline Opt Small Waist Right Height & 4 & 1.52 & -2.48 & 3.04 & -0.96 & 4.56 & 0.56 \\
\hline Half Back Centre & 5 & 2.06 & -2.94 & 4.12 & -0.88 & 6.18 & 1.18 \\
\hline Outside Leg Length (Right) & 4 & 1.61 & -2.39 & 3.21 & -0.79 & 4.82 & 0.82 \\
\hline Actual Ankle Circumference (Right) & 4 & 1.65 & -2.35 & 3.30 & -0.70 & 4.95 & 0.95 \\
\hline Elbow Circumference (Left) & 4 & 1.66 & -2.34 & 3.33 & -0.67 & 4.99 & 0.99 \\
\hline Outside Leg Length (Left) & 4 & 1.76 & -2.24 & 3.51 & -0.49 & 5.27 & 1.27 \\
\hline Across Back & 10 & 4.78 & -5.22 & 9.57 & -0.43 & 14.35 & 4.35 \\
\hline Actual Ankle Circumference (Left) & 4 & 1.84 & -2.16 & 3.67 & -0.33 & 5.51 & 1.51 \\
\hline Low Hip to Small Of Back Optimised Waist & 6 & 2.93 & -3.07 & 5.87 & -0.13 & 8.80 & 2.80 \\
\hline Actual Mid-Thigh Circumference (Right) & 6 & 3.00 & -3.00 & 6.01 & 0.01 & 9.01 & 3.01 \\
\hline
\end{tabular}




\begin{tabular}{|c|c|c|c|c|c|c|c|}
\hline Measurement & 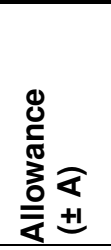 & $\stackrel{0}{-}$ & 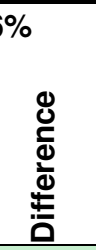 & $\stackrel{\mathfrak{D}}{\mathrm{N}}$ & 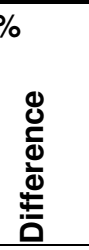 & $\underset{m}{b}$ & 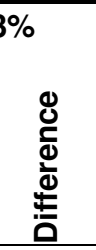 \\
\hline Actual Mid-Thigh Circumference Left & 6 & 3.01 & -2.99 & 6.02 & 0.02 & 9.03 & 3.03 \\
\hline Stomach Circumference & 11 & 5.65 & -5.35 & 11.30 & 0.30 & 16.95 & 5.95 \\
\hline Shoulder Length (Right) & 3 & 1.66 & -1.34 & 3.33 & 0.33 & 4.99 & 1.99 \\
\hline Wrist Circumference (Left) & 3 & 1.71 & -1.29 & 3.43 & 0.43 & 5.14 & 2.14 \\
\hline Min lower leg Height (Left) & 3 & 1.78 & -1.22 & 3.55 & 0.55 & 5.33 & 2.33 \\
\hline Front Hip & 7 & 3.78 & -3.22 & 7.57 & 0.57 & 11.35 & 4.35 \\
\hline Sleeve Length (Right) & 6 & 3.29 & -2.71 & 6.58 & 0.58 & 9.87 & 3.87 \\
\hline Back Neck to Back Chest Contour Length & 4 & 2.30 & -1.70 & 4.60 & 0.60 & 6.90 & 2.90 \\
\hline Arm Length (Right) & 6 & 3.30 & -2.70 & 6.61 & 0.61 & 9.91 & 3.91 \\
\hline Arm Under Length (Right) & 6 & 3.35 & -2.65 & 6.70 & 0.70 & 10.06 & 4.06 \\
\hline Abdomen Rise & 6 & 3.38 & -2.62 & 6.76 & 0.76 & 10.14 & 4.14 \\
\hline Back Shoulder Width Horizontal & 8 & 4.47 & -3.53 & 8.95 & 0.95 & 13.42 & 5.42 \\
\hline Shoulder Length (Left) & 3 & 1.98 & -1.02 & 3.97 & 0.97 & 5.95 & 2.95 \\
\hline Abdomen Height & 4 & 2.55 & -1.45 & 5.10 & 1.10 & 7.65 & 3.65 \\
\hline Min lower leg Height (Right) & 3 & 2.12 & -0.88 & 4.24 & 1.24 & 6.35 & 3.35 \\
\hline Front Waist & 6 & 3.66 & -2.34 & 7.32 & 1.32 & 10.98 & 4.98 \\
\hline Wrist Circumference (Right) & 3 & 2.28 & -0.72 & 4.56 & 1.56 & 6.84 & 3.84 \\
\hline Upper Bust Front Length & 8 & 4.89 & -3.11 & 9.78 & 1.78 & 14.67 & 6.67 \\
\hline Actual Knee Circumference (Left) & 4 & 3.43 & -0.57 & 6.87 & 2.87 & 10.30 & 6.30 \\
\hline Arm Length (Left) & 6 & 4.64 & -1.36 & 9.29 & 3.29 & 13.93 & 7.93 \\
\hline Actual Knee Circumference (Right) & 4 & 4.20 & 0.20 & 8.40 & 4.40 & 12.60 & 8.60 \\
\hline Sleeve Length (Left) & 6 & 6.39 & 0.39 & 12.79 & 6.79 & 19.18 & 13.18 \\
\hline Arm Under Length (Left) & 6 & 6.45 & 0.45 & 12.89 & 6.89 & 19.34 & 13.34 \\
\hline
\end{tabular}

\section{Discussion}

As Table 1 shows, less than half (49\%) of measurement taken by a 3D Body Scanner are suitable for garment construction purposes at a confidence level of $99.73 \%$. However, $51 \%$ of 3D Body Scanning measurements fall outside the allowable error for garment construction, though often by small amounts. This suggests that reliability is influenced by the location of measurements and raises the need to understand if all measurements give a reliable basis from which to create body worn products. This becomes difficult as few studies if any have addressed measurement repeatability by those producing clothing patterns, although this is further discussed within these proceedings [19].

While earlier studies have referred to 3D Body Scanning as being accurate [8], [11], [12], [16], this study is the first academic investigation into the reliability of the technology against allowable error from the best available guidance. In contrast to the earlier research and technical documents [27] that represents the technology as having a single answer of reliable to $\pm \chi \mathrm{mm}$, the reality is that 3D Body Scanning's reliability is relative to the measurement being taken and the application it is being applied to. Although previous research [4], [12], [15] has asserted that the reliability of 3D Body Scanning measurements are comparable to that of manual measuring methods. Assuming correct manual measurement techniques are taken, they may extract measurements to a reliability of between $\pm 3 \mathrm{~mm}$ up to $\pm 25 \mathrm{~mm}$ [14] and in some cases these may not be the largest recorded difference during the process. As a consequence, while 3D Body Scanning does not suffer from the influence of mistakes and human error [25], some measurements may not achieve suitable reliability against existing guidance, however, this does raise questions regarding reliability of measurements used in current clothing production practice. Often in pattern literature measurement guidance is scant, and unlike scanning, those being measured 
may adjust their posture within and between measurements [28]. This study shows that in some cases measurements may not meet scientific requirements of reliability, though as current practices in the industry are seldom studied, it is difficult to know how scan measurements may compare.

\section{Conclusion}

The main contribution of this study is that reliability and 3D body scanning is not only relative to the physical machine and sophistication of the analysis software, but also the specific body measurement being taken. This is the first investigation into the reliability of 3D Body Scanning against established allowable errors from scientific studies available to the public. This paper provides a tool in the form of a reliability assessment guide (Table 1) that any person may use to assess if 3D Body Scanning is a suitably reliable method for collecting body measurements for their specific need.

Managerial implications for garment manufacturers and ergonomists who wish to use 3D Body Scanning needs to be careful which measurement they are requiring to assess the reliability of their attained measurement. This is important for manufacturers of garments with higher measurement tolerance than utilised within this study, for example high compression sports garments. In such cases, where the reliability of 3D Body Scanning cannot be assured, traditional measurement methods (such as tape measures) should be considered the most reliable method of anthropometric measurement.

For scientific research, measurement purposes are however different since they are not working to manufacturing tolerances, but instead focus on comparing the difference between groups with statistics [2]. Because of this, scientists seeking to use 3D Body Scanning as an anthropometric tool must consult Table 1 when considering statistical outputs to decide if the between group differences are genuine (greater than error possible from reliability), or a Type I error (lower than error possible from reliability). This also raises the need to consider whether single scans offer data which can meet expert measurer accuracy, or whether the body scanner should look to create measurement lists from composite scan data removing outliers.

Limitations for this study stem from the limited sample number allowing for limited exploration of reliability. Besides this, the sample was mostly white (Caucasian), which may account for a limitation in anthropometric variation due to ethnicity. Additionally, only one 3D Body Scanner was used in the data collection, which limits the generalizability of the research to the Size Stream platform and those that also use comparable technology in the capture and analysis of the data.

Further research focussing on an increased spectrum of data from multiple 3D Body Scanners (e.g. $\mathrm{TC}^{2}$, Human Solutions) is required to give a more comprehensive assess and assert of the reliability of 3D Body Scanning. Future research needs to develop logical processing algorithms for software to show when multiple measurements produce a variation outside the allowable error and remove extreme values where suitable. Further research is also required on the influence of independent variables such as waist-to-height ratio as an expression of body fat, and ethnicity to predict the extent to which 3D Body Scanning might summarise the body to support its analysis. In addition to this, further development is required from the manufacturers relating to the measurement capture technology and data analysis to increase the reliability of the measurements being captured. There is also a clear need to determine what repeatability might be achieved by current industry practitioners to understand how realistic the allowable errors are from scientific surveys and how they compare to data used to drive product development.

\section{References}

[1] E. P. DeGarmo, J. T. Black, and R. A. Kohser, Materials and Processes in Manufacturing, 9th ed., vol. Ninth. Danvers, USA: John Wiley \& Sons, 2003.

[2] B. G. Tabachnick and L. S. Fidell, Using Multivariate Statistics, 4th ed. Needham Heights: Allyn and Bacon, 2001.

[3] K. McCarthy, B. Perkins, N. Pope, L. Portaluppi, V. Scaramuzzi, and L. Su, "Global Powers of Luxury Goods 2017," Westlake, USA, 2017.

[4] S. Gill and C. J. Parker, "Scan posture definition and hip girth measurement: the impact on clothing design and body scanning," Ergonomics, vol. 60, no. 8, pp. 1123-1136, Aug. 2017.

[5] L. Chi and R. Kennon, "Body scanning of dynamic posture," Int. J. Cloth. Sci. Technol., vol. 18, no. 3, pp. 166-178, 2006. 
[6] M. Januszkiewicz, C. J. Parker, S. G. Hayes, and S. Gill, "Online Virtual Fit Has Yet To Exploit It's Potential: Exploratory analysis of online virtual try-on interfaces," in 8th International Conference and Exhibition on 3D Body Scanning and Processing Technologies, 2017.

[7] E. Bye, K. L. LaBat, and M. R. Delong, "Analysis of Body Measurement Systems for Apparel," Cloth. Text. Res. J., vol. 24, no. 2, pp. 66-79, 2006.

[8] C. L. Istook and S.-J. Hwang, "3D body scanning systems with application to the apparel industry," J. Fash. Mark. Manag., vol. 5, no. 2, pp. 120-132, 2001.

[9] J. Park, Y. Nam, E. Lee, and S. Park, "Error detection in three-dimensional surface anthropometric data," Int. J. Ind. Ergon., vol. 39, no. 1, pp. 277-282, 2009.

[10] S. J. Ulijaszek and D. A. Kerr, "Anthropometric measurement error and the assessment of nutritional status," Br. J. Nutr., vol. 82, no. 3, pp. 165-177, Mar. 1999.

[11] K. P. Simmons and C. L. Istook, "Body measurement techniques: Comparing 3D body-scanning and anthropometric methods for apparel applications," J. Fash. Mark. Manag. An Int. J., vol. 7, no. 3, pp. 306-332, Sep. 2003.

[12] J. P. Bougourd, L. Dekker, P. Grant Ross, and J. P. Ward, "A Comparison of Women's Sizing by 3D Electronic Scanning and Traditional Anthropometry," J. Text. Inst., vol. 91, no. 2, pp. 163173, 2000.

[13] D.-E. Kim, K. LaBat, E. Bye, M. Sohn, and K. Ryan, "A study of scan garment accuracy and reliability," J. Text. Inst., vol. 106, no. 8, pp. 853-861, 2015.

[14] C. C. Gordon et al., "2012 Anthropometric Survey of U.S. Army Personnel: Methods and Summary Statistics," Massachusetts, USA, 2012.

[15] A. Kuehnapfel, P. Ahnert, M. Loeffler, A. Broda, and M. Scholz, "Reliability of 3D laser-based anthropometry and comparison with classical anthropometry," Sci. Rep., vol. 6, no. 1, p. 26672, Jul. 2016.

[16] H. Han, Y. Nam, and K. Choi, "Comparative analysis of 3D body scan measurements and manual measurements of size Korea adult females," Int. J. Ind. Ergon., vol. 40, no. 5, pp. 530540, Sep. 2010.

[17] S. Gill, C. J. Parker, S. Hayes, P. Wren, and A. Panchenko, "The True Height of the Waist: Explorations of automated body scanner waist definitions of the TC2 scanner," in 5th International Conference and Exhibition on 3D Body Scanning Technologies, 2014, pp. 55-65.

[18] ISO 5725-1, "Accuracy (trueness and precision) of measurement methods and results-Part 1: General principles and definitions," Geneva, Switzerland, 1998.

[19] S. Gill, M. Ahmed, C. J. Parker, and S. G. Hayes, "Not All Body Scanning Measurements Are Valid: Perspectives from Pattern Practice," in 8th International Conference and Exhibition on 3D Body Scanning and Processing Technologies, 2017.

[20] S. Gill, S. Hayes, and C. J. Parker, "3D Body Scanning: Towards a Shared Protocol," in IWAMA 2016: 6th International Workshop of Advanced Manufacturing and Automation, 2016, pp. 281284.

[21] C. C. Gordon et al., "1987-1988 Anthropometric Survey of U.S. Army Personnel: Methods and Summary Statistics. Technical Report (TR-89-044) (AD A225 094)," Natick, USA, 1989.

[22] BSI, "BS EN ISO 20685:2010: 3-D scanning methodologies for internationally compatible anthropometric databases," British Standards Institute, London, UK, 2010.

[23] SizeStream, "SS14 3D Body Scanner." SizeStream, Cary, NC, USA, 2017.

[24] IBM, "SPSS," vol. 23. IBM Ltd., USA, 2015.

[25] M. Pentz and M. Shott, Handling Experimental Data. Milton Keynes, UK: Open University Press, 1988.

[26] J. W. Tukey, Exploratory Data Analysis. Boston, USA: Addison-Wesley Publishing Company, 1977.

[27] TC2, "[TC]2," vol. 7.2. TC2, Cary, NC, USA, 2011.

[28] S. Gill, "A review of research and innovation in garment sizing, prototyping and fitting," Text. Prog., vol. 47, no. 1, pp. 1-85, Jan. 2015. 